\title{
Label Placement in Road Maps
}

\author{
Andreas Gemsa, Benjamin Niedermann, and Martin Nöllenburg \\ Institute of Theoretical Informatics, Karlsruhe Institute of Technology, Germany
}

\begin{abstract}
A road map can be interpreted as a graph embedded in the plane, in which each vertex corresponds to a road junction and each edge to a particular road section. We consider the cartographic problem to place non-overlapping road labels along the edges so that as many road sections as possible are identified by their name, i.e., covered by a label. We show that this is NP-hard in general, but the problem can be solved in polynomial time if the road map is an embedded tree.
\end{abstract}

\section{Introduction}

Map labeling is a well-known cartographic problem in computational geometry [11, Chapter 58.3.1], [13]. Depending on the type of map features, one can distinguish labeling of points, lines, and areas. Common cartographic quality criteria are that labels must be disjoint and clearly identify their respective map features [7]. Most of the previous work concerns point labeling, while labeling line and area features received considerably less attention. In this paper we address labeling linear features, namely roads in a road map.

Geometrically, a road map is the representation of a road $\operatorname{graph} G$ as an arrangement of fat curves in the plane $\mathbb{R}^{2}$. Each road is a connected subgraph of $G$ (typically a simple path) and each edge belongs to exactly one road. Roads may intersect each other in junctions, the vertices of $G$, and we denote an edge connecting two junctions as a road section. In road labeling the task is to place the road names inside the fat curves so that the road sections are identified unambiguously, see Fig. 1 .

Chirié [1] presented a set of rules and quality criteria for label placement in road maps based on interviews with cartographers. This includes that (C1) labels are placed inside and parallel to the road shapes, (C2) every road section between two junctions should be clearly identified, and (C3) no two road labels may intersect. Further, he gave a mathematical description for labeling a single road and introduced a heuristic for sequentially labeling all roads in the map. Imhof's foundational cartographic work on label positioning in maps lists very similar quality criteria [3]. Edmondson et al. [2] took an algorithmic perspective on labeling a single linear feature (such as a river). While Edmondson et al. considered non-bent labels, Wolff et al. [12] introduced an algorithm for single linear feature that places labels following the curvature of the linear feature. Strijk [9] considered static road labeling with embedded labels and presented a heuristic for selecting non-overlapping labels out of a set of label candidates. Seibert and Unger $[8]$ considered grid-shaped road networks. They showed that in those networks it is NP-complete and APX-hard to decide whether for every road at least one label can be placed. Yet, Neyer and Wagner [6] introduced a practically efficient algorithm that 

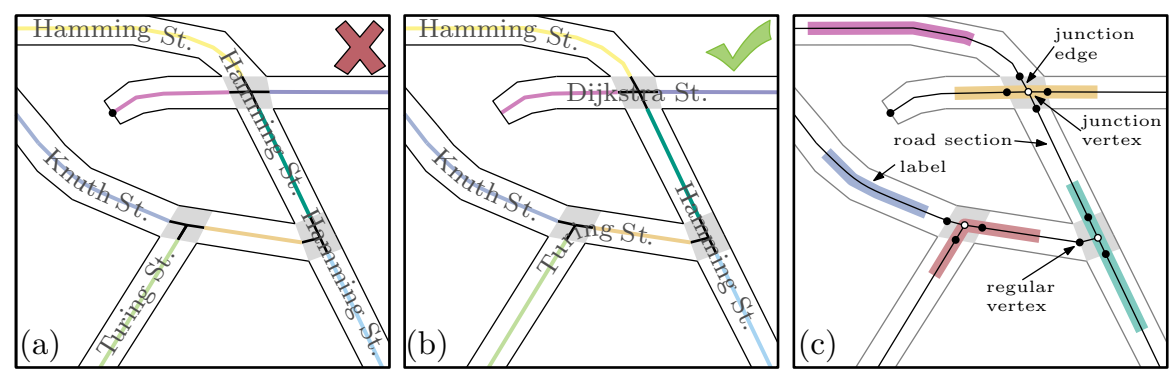

Fig. 1. a-b): Two ways to label the same road network. Each road section has its own color. Junctions are marked gray. Fig. b) identifies all road sections. c) Illustration of the road graph and relevant terms.

finds such a grid labeling if possible. Maass and Döllner [5] presented a heuristic for labeling the roads of an interactive 3D map with objects (such as buildings). Apart from label-label overlaps, they also resolve label-object occlusions. Vaaraniem et al. [10] used a force-based labeling algorithm for 2D and 3D scenes including road label placement.

Contribution. While in grid-shaped road networks it is sufficient to place a single label per road to clearly identify all its road sections, this is not the case in general road networks. Consider the example in Fig. 1. In Fig. (17), it is not obvious whether the orange road section in the center belongs to Knuth St. or to Turing St. Simply maximizing the number of placed labels, as often done for labeling point features, can cause undesired effects like unnamed roads or clumsy label placements (e.g., around Dijkstra St. and Hamming St. in Fig. (19)). Therefore, in contrast to Seibert and Unger [8], we aim for maximizing the number of identified road sections, i.e., road sections that can be clearly assigned to labels; see Fig. 1p).

Based on criteria (C1)-(C3) we introduce a new and versatile model for road labeling in Section 2. In Section 3 we show that the problem of maximizing the number of identified road sections is NP-hard for general road graphs, even if each road is a path. For the special case that the road graph is a tree, we present a polynomial-time algorithm in Section 4 This special case is not only of theoretical interest, but our algorithm in fact provides a very useful subroutine in exact or heuristic algorithms for labeling general road graphs. Our initial experiments, sketched in Section 5, show that real-world road networks decompose into small subgraphs, a large fraction of which (more than $85.1 \%$ ) are actually trees, and thus can be labeled optimally by our algorithm.

\section{Preliminaries}

As argued above, a road map is a collection of fat curves in the plane, each representing a particular piece of a named road. If two (or more) such curves intersect, they form junctions. A road label is again a fat curve (the bounding shape of the road name) that is contained in and parallel to the fat curve representing its road. We observe that labels of different roads can intersect only within junctions and that the actual width of the curves 
is irrelevant, except for defining the shape and size of the junctions. These observations allow us to define the following more abstract but equivalent road map model.

A road map $\mathcal{M}$ is a planar road graph $G=(V, E)$ together with a planar embedding $\mathrm{E}(G)$, which can be thought of as the geometric representation of the road axes as thin curves; see Fig 1 1 ). We denote the number of vertices of $G$ by $n$, and the number of edges by $m$. Observe that since $G$ is planar $m=O(n)$. Each edge $e \in E$ is either a road section, which is not part of a junction, or a junction edge, which is part of a junction. Each vertex $v \in V$ is either a junction vertex incident only to junction edges, or a regular vertex incident to one road section and at most one junction edge, which implies that each regular vertex has degree at most two. A junction vertex $v$ and its incident edges are denoted as a junction. The edge set $E$ decomposes into a set $\mathcal{R}$ of edge-disjoint roads, where each road $R \in \mathcal{R}$ induces a connected subgraph of $G$. Without loss of generality we assume no two road sections $G$ are incident to the same vertex. Thus, a road decomposes into road sections, separated by junction vertices and their incident junction edges. In realistic road networks the number of roads connected passing through a junction is small and does not depend on the size of the road network. We therefore assume that each vertex in $G$ has constant degree. We assume that each road $R \in \mathcal{R}$ has a name whose length we denote by $\lambda(R)$.

For simplicity, we identify the embedding $\mathrm{E}(G)$ with the points in the plane covered by $\mathrm{E}(G)$, i.e. $\mathrm{E}(G) \subseteq \mathbb{R}^{2}$. We also use $\mathrm{E}(v), \mathrm{E}(e)$, and $\mathrm{E}(R)$ to denote the embeddings of a vertex $v$, an edge $e$, and a road $R$.

We model a label as a simple open curve $\ell:[0,1] \rightarrow \mathrm{E}(G)$ in $\mathrm{E}(G)$. Unless mentioned otherwise, we consider a curve $\ell$ always to be simple and open, i.e., $\ell$ has no self-intersections and its end points do not coincide. In order to ease the description, we identify a curve $\ell$ in $\mathrm{E}(G)$ with its image, i.e., $\ell$ denotes the set $\{\ell(t) \in \mathrm{E}(G) \mid t \in[0,1]\}$. The start point of $\ell$ is denoted as the head $h(\ell)$ and the endpoint as the tail $t(\ell)$. The length of $\ell$ is denoted by len $(\ell)$. The curve $\ell$ identifies a road section $r$ if $\ell \cap \mathrm{E}(r) \neq \emptyset$. For a set $\mathcal{L}$ of curves $\omega(\mathcal{L})$ is the number of road sections that are identified by the curves in $\mathcal{L}$. For a single curve $\ell$ we use $\omega(\ell)$ instead of $\omega(\{\ell\})$. For two curves $\ell_{1}$ and $\ell_{2}$ it is not necessarily true that $\omega\left(\left\{\ell_{1}, \ell_{2}\right\}\right)=\omega\left(\ell_{1}\right)+\omega\left(\ell_{2}\right)$, because they may identify the same road section twice.

A label $\ell$ for a road $R$ is a curve $\ell \subseteq \mathrm{E}(R)$ of length $\lambda(R)$ whose endpoints must lie on road sections and not on junction edges or junction vertices. Requiring that labels end on road sections avoids ambiguous placement of labels in junctions where it is unclear how the road passes through it. A labeling $\mathcal{L}$ for a road map with road set $\mathcal{R}$ is a set of mutually non-overlapping labels, where we say that two labels $\ell$ and $\ell^{\prime}$ overlap if they intersect in a point that is not their respective head or tail.

Following the cartographic quality criteria $(\mathrm{C} 1)-(\mathrm{C} 3)$, our goal is to find a labeling $\mathcal{L}$ that maximizes the number of identified road sections, i.e., for any labeling $\mathcal{L}^{\prime}$ we have $\omega\left(\mathcal{L}^{\prime}\right) \leq \omega(\mathcal{L})$. We call this problem MAXIDENTIFIEDRoAds.

Note that assuming the road graph $G$ to be planar is not a restriction in practice. Consider for example a road section $r$ that overpasses another road section $r^{\prime}$, i.e., $r$ is a bridge over $r^{\prime}$, or $r^{\prime}$ is a tunnel underneath $r$. In order to avoid overlaps between labels placed on $r$ and $r^{\prime}$, we either can model the intersection of $r$ and $r^{\prime}$ as a regular crossing of two roads or we split $r^{\prime}$ in smaller road sections that do not cross $r$. In both cases 


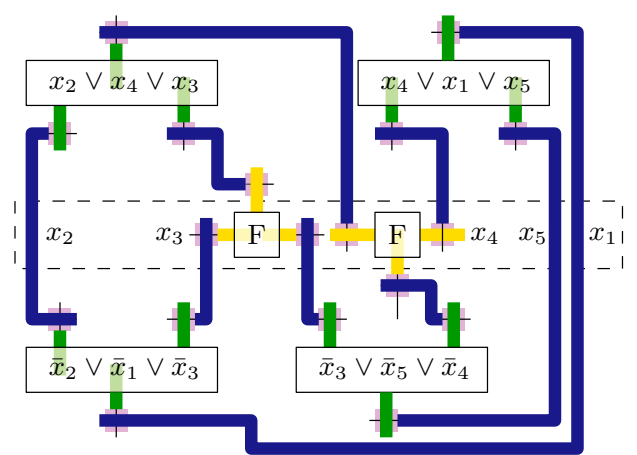

(a) Sketch of $\mathcal{M}_{\varphi}$

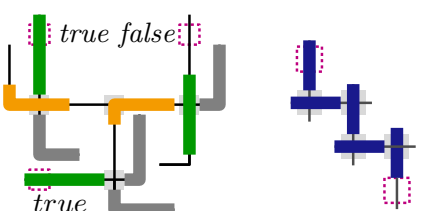

(c) Chain
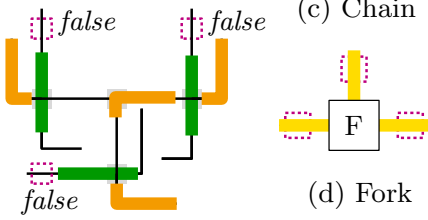

(d) Fork

(b) Clause

Fig. 2. Illustration of NP-hardness proof. (a) 3-Sat formula $\varphi=\left(x_{4} \vee x_{1} \vee x_{5}\right) \wedge\left(x_{2} \vee x_{4} \vee\right.$ $\left.x_{3}\right) \wedge\left(\bar{x}_{2} \vee \bar{x}_{1} \vee \bar{x}_{3}\right) \wedge\left(\bar{x}_{3} \vee \bar{x}_{5} \vee \bar{x}_{4}\right)$ represented as road graph $\mathcal{M}_{\varphi}$. Truth assignment is $x_{1}=$ true, $x_{2}=$ true, $x_{3}=$ false, $x_{4}=$ false and $x_{5}=$ false. (b) Clause gadget in two states. (c) The chain is the basic building block for the proof. (d) Schematized fork gadget.

the corresponding road graph becomes planar. In the latter case we may obtain more independent roads created by chopping $r^{\prime}$ into smaller pieces.

\section{Computational Complexity}

We first study the computational complexity of road labeling and prove NP-hardness of MAXIDENTIFIEDROADS in the following sense.

Theorem 1. For a given road map $\mathcal{M}$ and an integer $K$ it is NP-hard to decide if in total at least $K$ road sections can be identified.

Proof. We perform a reduction from the NP-complete PLANAR MONOTONE 3-SAT problem [4]. An instance of PLANAR MONOTONE 3-SAT is a Boolean formula $\varphi$ with $n$ variables and $m$ clauses (disjunctions of at most three literals) that satisfies the following additional requirements: (i) $\varphi$ is monotone, i.e., every clause contains either only positive literals or only negative literals and (ii) the induced variable-clause graph $H_{\varphi}$ of $\varphi$ is planar and can be embedded in the plane with all variable vertices on a horizontal line, all positive clause vertices on one side of the line, all negative clauses on the other side of the line, and the edges drawn as rectilinear curves connecting clauses and contained variables on their respective side of the line. We construct a road map $\mathcal{M}_{\varphi}$ that mimics the shape of the above embedding of $H_{\varphi}$ by defining variable and clause gadgets, which simulate the assignment of truth values to variables and the evaluation of the clauses. We refer to Fig. 2 for a sketch of the construction.

Chain Gadget. The basic building block is the chain gadget, which consists of an alternating sequence of equally long horizontal and vertical roads with identical label lengths that intersect their respective neighbors in the sequence and form junctions with 


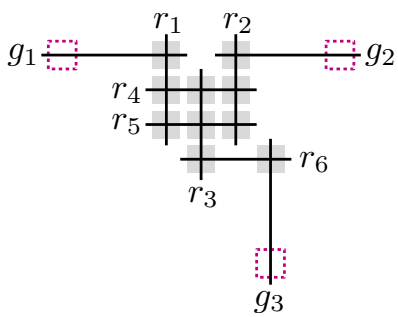

(a)

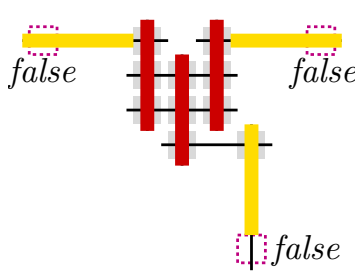

(b)

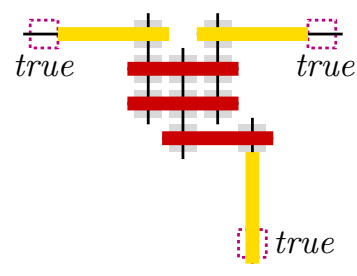

(c)

Fig. 3. Illustration of the fork gadget.(a) Structure of the fork gadget.(c) Configuration transmitting the value false.(b) Configuration transmitting the value true.

them as indicated in Fig. 2k). Assume that the chain consists of $k \geq 3$ roads. Then each road except the first and last one decomposes into three road sections split by two junctions, a longer central section and two short end sections; the first and last road consist of only two road sections, a short one and a long one, separated by one junction. (These two roads will later be connected to other gadgets; indicated by dotted squares in Fig. 2k).) The label length and distance between junctions is chosen so that for each road either the central and one end section is identified, or no section at all is identified. For the first and last road, both sections are identified if the junction is covered and otherwise only the long section can be identified. We have $k$ roads and $k-1$ junctions. Each label must block a junction, if it identifies two sections. So the best possible configuration blocks all junctions and identifies $2(k-1)+1=2 k-1$ road sections.

The chain gadget has exactly two states, in which $2 k-1$ road sections are identified. Either the label of the first road does not block a junction and identifies a single section and all subsequent roads have their label cover the junction with the preceding road in the sequence, or the label of the last road does not block a junction and all other roads have their label cover the junction with the successive road in the sequence. In any other configuration there is at least one road without any identified section and thus at most $2 k-2$ sections are identified. We use the two optimal states of the gadget to represent and transmit the values true and false from one end to the other.

Fork Gadget. The fork gadget allows to split the value represented in one chain into two chains, which is needed to transmit the truth value of a variable into multiple clauses. To that end it connects to an end road of three chain gadgets by sharing junctions.

The core of the fork consists of six roads $r_{1}, \ldots, r_{6}$, whereas $r_{1}, r_{2}$, and $r_{3}$ are vertical line segments and $r_{4}, r_{5}$ and $r_{6}$ are horizontal line segments; see Fig. 3 We arrange those roads such that $r_{1}$ and $r_{2}$ have each one junction with $r_{4}$ and one junction with $r_{5}$. Further, $r_{3}$ has one junction with $r_{4}$, one with $r_{5}$ and one with $r_{6}$. The label length of those roads is chosen so that it is exactly the length of the roads. Hence, a placed label idenfies all road sections of the roads.

Further, there are three roads $g_{1}, g_{2}, g_{3}$ such that $g_{1}$ has one junction with $r_{1}, g_{2}$ has one junction with $r_{2}$ and $g_{3}$ has one junction with $r_{6}$. In all three cases we place the junction so that it splits the road in a short road section that is shorter than the road's label length and a long road section that has exactly the road's label length. We call $g_{1}$, 
$g_{2}$ and $g_{3}$ gates, because later these roads will be connected to the end roads of chains by junctions. To that end those connecting junctions will be placed on the long road sections of the gates; see violet dotted areas in Fig. 3 .

The fork gadget has exactly two states, in which 16 road sections are identified. In the first state the labels of $r_{1}, r_{2}$ and $r_{3}$ are placed; see Fig 3(b). Hence, the labels of $g_{1}$ and $g_{2}$ identify only the long road sections of $g_{1}$ and $g_{2}$, but not the short ones. The label of $g_{3}$ idenfies both the long and short road section of $g_{3}$. In the second state the labels of $r_{4}, r_{5}, r_{6}$ are placed; see Fig 3(c). Hence, the labels of $g_{1}$ and $g_{2}$ identify the long and short road sections of $g_{1}$ and $g_{2}$, while only the long road section of $g_{3}$ is identified by a label. In any other configuration fewer road sections are identified by labels. We use the two optimal states of the gadget to represent and transmit the values true and false from one gate to the other two gates. More specifically the gates $g_{1}$ and $g_{2}$ are connected with chains that lead to the same literal, while $g_{3}$ is connected with a chain that leads to the complementary literal.

Variable Gadget. We define the variable gadgets simply by connecting chain and fork gadgets into a connected component of intersecting roads. This construction already has the functionality of a variable gadget: it represents (in a labeling identifying the maximum number of road sections) the same truth value in all of its branches, synchronized by the fork gadgets, see the blue chains and yellow forks in Fig. 2a). More precisely, we place a sequence of chains linked by fork gadgets along the horizontal line on which the variable vertices are placed in the drawing $H_{\varphi}$. Each fork creates a branch of the variable gadget either above or below the line. We create as many branches above (below) the line as the variable has occurrences in positive (negative) clauses in $\varphi$. The first and last chain on the line also serve as branches. The synchronization of the different branches via the forks is such that either all top branches have their road labels pushed away from the line and all bottom branches pulled towards the line or vice versa. In the first case, we say that the variable is in the state false and in the latter case that it is in the state true. The example in Fig. 2 2 has two variables set to true and three variables set to false.

Clause Gadget. Finally, we need to create the clause gadget, which links three branches of different variables. The core of the gadget is a single road that consists of three sub-paths meeting in one junction. Each sub-path of that road shares another junction with one of the three incoming variable branches. Beyond each of these three junctions the final road sections are just long enough so that a label can be placed on the section. However, the section between the central junction of the clause road and the junctions with the literal roads is shorter than the label length. The road of the clause gadget has six sections in total and we argue that the six sections can only be identified if at least one incoming literal evaluates to true. Otherwise at most five sections can be identified. By construction, each road in the chain of a false literal has its label pushed towards the clause, i.e., it blocks the junction with the clause road. As long as at least one of these three junctions is not blocked, all sections can be identified; see Fig. 22p). But if all three junctions are blocked, then only two of the three inner sections of the clause road can be identified and the third one remains unlabeled; see Fig. 20).

Reduction. Obviously, the size of the instance $\mathcal{M}_{\varphi}$ is polynomial in $n$ and $m$. If we have a satisfying variable assignment for $\varphi$, we can construct the corresponding road labeling and the number of identified road sections is six per clause and a fixed constant 
number $K^{\prime}$ of sections in the variable gadgets, i.e., at least $K=K^{\prime}+6 m$. On the other hand, if we have a road labeling with at least $K$ identified sections, each variable gadget is in one of its two maximum configurations and each clause road has at least one label that covers a junction with a literal road, meaning that the corresponding truth value assignment of the variables is indeed a satisfying one. This concludes the reduction.

Since MAXIdenTIFIEDRoADS is an optimization problem, we only present the NPhardness proof. Still, one can argue that the corresponding decision problem is NPcomplete by guessing which junctions are covered by which label and then using linear programming for computing the label positions. We omit the technical details. Further, most roads in the reduction are paths, except for the central road in each clause gadget, which is a degree-3 star. In fact, we can strengthen Theorem 1 by using a more complex clause gadget instead that uses only paths; see Appendix A.1

\section{An Efficient Algorithm for Tree-Shaped Road Maps}

In this section we assume that the underlying road graph of the road map is a tree $T=(V, E)$. In Section 4.1 we present a polynomial-time algorithm to optimally solve
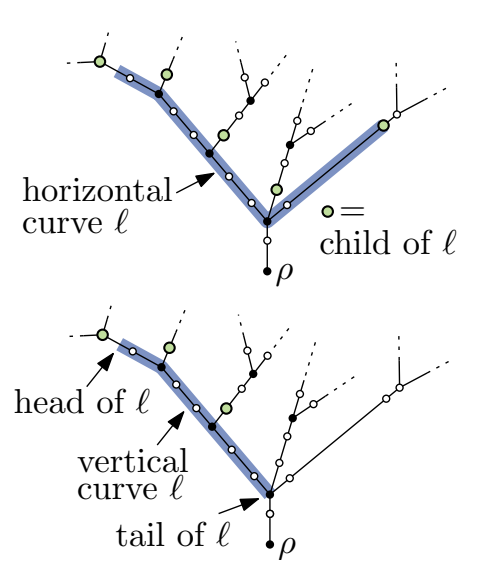

Fig. 4. Basic definitions. MAXIDENTIFIEDROADs for trees; Section 4.2 shows how to improve its running time and space consumption. Our approach uses the basic idea that removing the vertices, whose embeddings lie in a curve $c \subseteq \mathrm{E}(T)$, splits the tree into independent parts. In particular this is true for labels. We assume that $T$ is rooted at an arbitrary leaf $\rho$ and that its edges are directed away from $\rho$; see Fig. 4. For two points $p, q \in \mathrm{E}(T)$ we define $\mathrm{d}(p, q)$ as the length of the shortest curve in $\mathrm{E}(T)$ that connects $p$ and $q$. For two vertices $u$ and $v$ of $T$ we also write $\mathrm{d}(u, v)$ instead of $\mathrm{d}(\mathrm{E}(u), \mathrm{E}(v))$. For a point $p \in E(T)$ we abbreviate the distance $\mathrm{d}(p, \rho)$ to the root $\rho$ by $\mathrm{d}_{p}$. For a curve $\ell$ in $\mathrm{E}(T)$, we call $p \in \ell$ the lowest point of $\ell$ if $\mathrm{d}_{p} \leq \mathrm{d}_{q}$, for any $q \in \ell$. As $T$ is a tree, $p$ is unique. We distinguish two types of curves in $\mathrm{E}(T)$. A curve $\ell$ is vertical if $h(\ell)$ or $t(\ell)$ is the lowest point of $\ell$; otherwise we call $\ell$ horizontal (see Fig. (4). Without loss of generality we assume that the lowest point of each vertical curve $\ell$ is its tail $t(\ell)$. Since labels are modeled as curves, they are also either vertical or horizontal. For a vertex $u \in V$ let $T_{u}$ denote the subtree rooted at $u$.

\subsection{Basic Approach}

We first determine a finite set of candidate positions for the heads and tails of labels, and transform $T$ into a tree $T^{\prime}=\left(V^{\prime}, E^{\prime}\right)$ by subdividing some of $T$ 's edges so that it contains a vertex for every candidate position. To that end we construct for each regular vertex $v \in V$ a chain of tightly packed vertical labels that starts at $\mathrm{E}(v)$, is directed 
towards $\rho$, and ends when either the road ends, or adding the next label does not increase the number of identified road sections. More specifically, we place a first vertical label $\ell_{1}$ such that $h\left(\ell_{1}\right)=\mathrm{E}(v)$. For $i=2,3, \ldots$ we add a new vertical label $\ell_{i}$ with $h\left(\ell_{i}\right)=$ $t\left(\ell_{i-1}\right)$, as long as $h\left(\ell_{i}\right)$ and $t\left(\ell_{i}\right)$ do not lie on the same road section and none of $\ell_{i}$ 's endpoints lie on a junction edge. We use the tails of all those labels to subdivide the tree $T$. Doing this for all regular vertices of $T$ we obtain the tree $T^{\prime}$, which we call the subdivision tree of $T$. The vertices in $V^{\prime} \backslash V$ are neither junction vertices nor regular vertices. Since each chain consists of $O(n)$ labels the cardinality of $V^{\prime}$ is $O\left(n^{2}\right)$. We call an optimal labeling $\mathcal{L}$ of $T$ an canonical labeling if for each label $\ell \in \mathcal{L}^{\prime}$ there exists a vertex $v$ in $T^{\prime}$ with $\mathrm{E}(v)=h(\ell)$ or $\mathrm{E}(v)=t(\ell)$. The next lemma proves that is sufficient to consider canonical labelings.

Lemma 1. For any road graph $T$ that is a tree, there exists a canonical labeling $\mathcal{L}$.

Proof. Let $\mathcal{L}$ be an optimal labeling of $T$. We push the labels of $\mathcal{L}$ as far as possible towards the leaves of $T$ without changing the identified road sections; see Fig. 5 More specifically, starting with the labels closest to the leaves, we move each label away from the root as far as possible while its head and tail must remain on their respective road sections. For a vertical label this direction is unique, while for horizontal labels we can choose any of the two. Then, for each label its head or tail either coincides with a leaf of $T$, with some internal regular vertex, or with the head of another label. Consequently, each vertical label belongs to a chain of tightly packed vertical labels starting at a regular vertex $v \in V$. Further, the head or tail of each horizontal label coincides with the end of a chain of tightly packed vertical labels or a regular vertex of $T$, which proves the claim.

We now explain how to construct such a canonical labeling. To that end we first introduce some notations. For a vertex $u \in V^{\prime}$ let $\mathcal{L}(u)$ denote a labeling that identifies

(a)
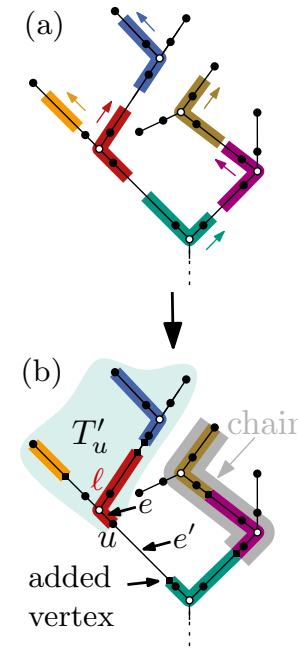

Fig. 5. Canonical labeling. a maximum number of road sections in $T$ only using valid labels in $\mathrm{E}\left(T_{u}^{\prime}\right)$, where $T_{u}^{\prime}$ denotes the subtree of $T^{\prime}$ rooted at $u$. Note that those labels also may identify the incoming road section of $u$, e.g., label $\ell$ in Fig. 5p) identifies the edge $e^{\prime}$.

Further, the children of a vertex $u \in V^{\prime}$ are denoted by the set $N(u)$; we explicitly exclude the parent of $u$ from $N(u)$. Further, consider an arbitrary curve $\ell$ in $\mathrm{E}(T)$ and let $\ell^{\prime}=$ $\ell \backslash\{t(\ell), h(\ell)\}$. We observe that removing all vertices of $T^{\prime}$ contained in $\ell^{\prime}$ together with their incident outgoing edges creates several independent subtrees. We call the roots of these subtrees (except the one containing $\rho$ ) children of $\ell$ (see Fig.44. If no vertex of $T^{\prime}$ lies in $\ell^{\prime}$, the curve is contained in a single edge $(u, v) \in E^{\prime}$. In that case $v$ is the only child of $\ell$. We denote the set of all children of $\ell$ as $N(\ell)$.

For each vertex $u$ in $T^{\prime}$ we introduce a set $C(u)$ of candidates, which model potential labels with lowest point $\mathrm{E}(u)$. If $u$ is a regular vertex of $T$ or $u \in V^{\prime} \backslash V$, the set $C(u)$ contains all vertical labels $\ell$ with lowest point $\mathrm{E}(u)$. If $u$ is a junction vertex, $C(u)$ contains all horizontal labels that start or end at a vertex of 
$T^{\prime}$ and whose lowest point is $\mathrm{E}(u)$. In both cases we assume that $C(u)$ also contains the degenerated curve $\perp_{u}=\mathrm{E}(u)$, which is the dummy label of $u$. We set $N\left(\perp_{u}\right)=N(u)$ and $\omega\left(\perp_{u}\right)=0$.

For a curve $\ell$ we define $\mathcal{L}(\ell)=\bigcup_{v \in N(\ell)} \mathcal{L}(v) \cup\{\ell\}$. Thus, $\mathcal{L}(\ell)$ is a labeling comprising $\ell$ and the labels of its children's optimal labelings. We call a label $\bar{\ell} \in C(u)$ with $\bar{\ell}=\operatorname{argmax}\{\omega(\mathcal{L}(\ell)) \mid \ell \in C(u)\}$ an optimal candidate of $u$. Next, we prove that it is sufficient to consider optimal candidates to construct a canonical labeling.

Lemma 2. Given a vertex $u$ of $T^{\prime}$ and an optimal labeling $\mathcal{L}(u)$ and let $\bar{\ell}$ be an optimal candidate of $u$, then it is true that $\omega(\mathcal{L}(u))=\omega(\mathcal{L}(\bar{\ell}))$.

Proof. First note that $\omega(\mathcal{L}(u)) \geq \omega(\mathcal{L}(\bar{\ell}))$ because both labelings $\mathcal{L}(u)$ and $\mathcal{L}(\bar{\ell})$ only contain labels that are embedded in $\mathrm{E}\left(T_{u}^{\prime}\right)$. By Lemma 1 we can assume without loss of generality that $\mathcal{L}(u)$ is a canonical labeling. Let $\ell$ be the label of $\mathcal{L}(u)$ with $\mathrm{E}(u)$ as the lowest point of $\ell$ (if it exists).

If $\ell$ exists, then the vertices in $N(\ell)$ are roots of independent subtrees, which directly yields $\omega(\mathcal{L}(u))=\omega(\mathcal{L}(\ell))$. By construction of $C(u)$ we further know that $\ell$ is contained in $C(u)$. Hence, $\ell$ is an optimal candidate of $u$, which implies $\omega(\ell)=\omega(\bar{\ell})$.

If $\ell$ does not exist, then we have

$$
\omega(\mathcal{L}(u))=\omega\left(\bigcup_{v \in N(u)} \mathcal{L}(v)\right) \stackrel{(1)}{=} \omega\left(\bigcup_{v \in N\left(\perp_{u}\right)} \mathcal{L}(v) \cup\left\{\perp_{u}\right\}\right)=\omega\left(\mathcal{L}\left(\perp_{u}\right)\right) .
$$

Equality (1) follows from $N\left(\perp_{u}\right)=N(u)$ and the definition that $\perp_{u}$ does not identify any road section. Since $\perp_{u}$ is contained in $C(u)$, the dummy label $\perp_{u}$ is the optimal candidate $\bar{\ell}$.

Algorithm 1 first constructs the subdivision tree $T^{\prime}=\left(V^{\prime}, E^{\prime}\right)$ from $T$. Then starting with the leaves of $T^{\prime}$ and going to the root $\rho$ of $T^{\prime}$, it computes an optimal candidate $\bar{\ell}=$ optCandidate $(u)$ for each vertex $u \in V^{\prime}$ in a bottom-up fashion. By Lemma 2 the labeling $\mathcal{L}(\bar{\ell})$ is an optimal labeling of $T_{u}^{\prime}$. In particular $\mathcal{L}(\rho)$ is the optimal labeling of $T$.

Due to the size of the subdivision tree $T^{\prime}$ we consider $O\left(n^{2}\right)$ vertices. Implementing OptCandidate $(u)$, which computes an optimal candidate $\bar{\ell}$ for $u$, naively, creates $C(u)$ explicitly. We observe that if $u$ is a junction vertex, $C(u)$ may contain $O\left(n^{2}\right)$ labels; $O\left(n^{2}\right)$ pairs of road sections of different subtrees of $u$ can be connected by

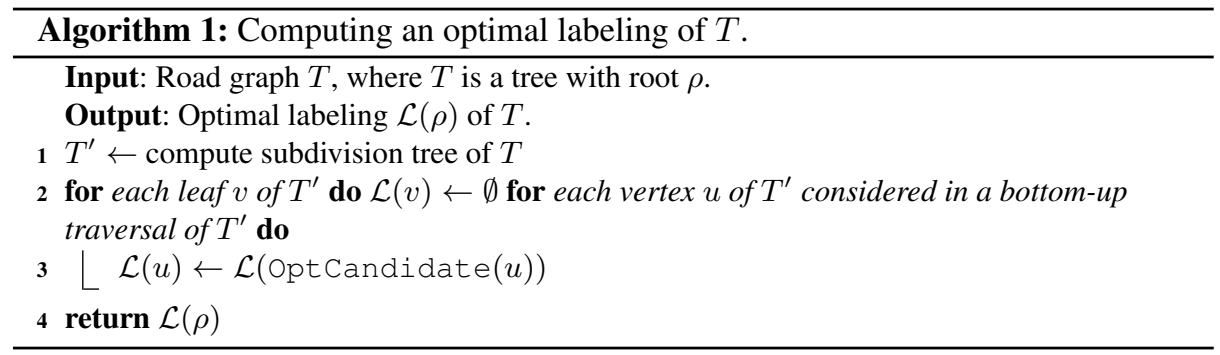


horizontal labels. Each label can be constructed in $O(n)$ time using a breadth-first search. Thus, for each vertex $u$ the procedure OptCandidate needs in a naive implementation $O\left(n^{3}\right)$ time, which yields $O\left(n^{5}\right)$ running time in total. Further, we need $O\left(n^{2}\right)$ storage to store $T^{\prime}$. Note that we do not need to store $\mathcal{L}(u)$ for each vertex $u$ of $T^{\prime}$, but by Lemma 2 we can reconstruct it using $\mathcal{L}(\bar{\ell})$, where $\bar{\ell}$ is the optimal candidate of $u$. To that end we store for each vertex of $T^{\prime}$ its optimal candidate $\bar{\ell}$ and $w(\mathcal{L}(\bar{\ell}))$.

Theorem 2. For a road map with a tree as underlying road graph, MAXIDENTIFIEDROADS can be solved in $O\left(n^{5}\right)$ time using $O\left(n^{2}\right)$ space.

In case that all roads are paths, Algorithm 1 runs in $O\left(n^{4}\right)$ time, because for each $u \in V^{\prime}$ the set $C(u)$ contains $O(n)$ labels. Further, besides the primary objective to identify a maximum number of road sections, Chirié [1] also suggested several additional secondary objectives, e.g., labels should overlap as few junctions as possible. Our approach allows us to easily incorporate secondary objectives by changing the weight function $\omega$ appropriately.

\subsection{Improvements on Running Time}

In this part we describe how the running time of Algorithm 1 can be improved to $O\left(n^{3}\right)$ time by speeding up OptCandidate $(u)$ to $O(n)$ time.

For an edge $e=(u, v) \in E \cup E^{\prime}$ we call a vertical curve $\ell \subseteq \mathrm{E}(T)$ an $e$-rooted curve, if $t(\ell)=\mathrm{E}(u), h(\ell)$ lies on a road section, and $\operatorname{len}(\mathrm{E}(e) \cap \ell)=\min \{\operatorname{len}(\ell), \operatorname{len}(\mathrm{E}(e))\}$, i.e., $\ell$ emanates from $\mathrm{E}(u)$ passing through $e$; for example the red label in Fig. 5p) is an $e$-rooted curve. An $e$-rooted curve $\ell$ is maximal if there is no other $e$-rooted curve $\ell^{\prime}$ with len $(\ell)=\operatorname{len}\left(\ell^{\prime}\right)$ and $\omega\left(\mathcal{L}\left(\ell^{\prime}\right)\right)>\omega(\mathcal{L}(\ell))$. We observe that in any canonical labeling each vertical label $\ell$ is a $(u, v)$-rooted curve with $(u, v) \in E^{\prime}$, and each horizontal label $\ell$ can be composed of a $\left(u, v_{1}\right)$-rooted curve $\ell_{1}$ and a $\left(u, v_{2}\right)$ rooted curve $\ell_{2}$ with $\left(u, v_{1}\right),\left(u, v_{2}\right) \in E^{\prime}$ and $\mathrm{E}(u)$ is the lowest point of $\ell$; see Fig. 7 and Fig. 8, respectively. Further, for a vertical curve $c$ in $\mathrm{E}(T)$ its distance interval $I(c)$ is $\left[\mathrm{d}_{t(c)}, \mathrm{d}_{h(c)}\right]$. Since $T$ is a tree, for every point $p$ of $c$ we have $\mathrm{d}_{p} \in I(c)$. Two vertical curves $c$ and $c^{\prime}$ superpose each other if $I(c) \cap I\left(c^{\prime}\right) \neq \emptyset$; see Fig 6 .

Next, we introduce a data structure that encodes for each edge $(u, v)$ of $T$ all maximal $(u, v)$-rooted curves as $O(n)$ superposition-free curves in $\mathrm{E}\left(T_{u}\right)$. In particular, each of those curves lies on a single road section such that all $(u, v)$-rooted curves ending on that curve are maximal and identify the same number of road sec-

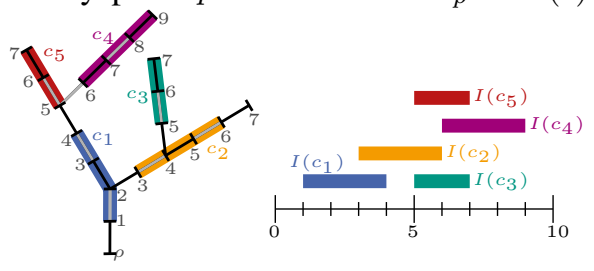

Fig. 6. Superposing curves, e.g., $c_{1}$ and $c_{2}$ superpose each other, while $c_{1}$ and $c_{5}$ do not. The tree is annotated with distance marks.

tions. We define this data structure as follows.

Definition 1 (Linearization). Let $e=(u, v)$ be an edge of $T$. A tuple $(L, \bar{\omega})$ is called a linearization of $e$, if $L$ is a set of superposition-free curves and $\bar{\omega}: L \rightarrow \mathbb{R}$ such that (1) for each curve $c \in L$ there is a road section $e^{\prime}$ in $T_{u}$ with $c \subseteq \mathrm{E}\left(e^{\prime}\right)$, 
(2) for each e-rooted curve $\ell$ there is a curve $c \in L$ with $\operatorname{len}(\ell)+\mathrm{d}_{u} \in I(c)$,

(3) for each point $p$ of each curve $c \in L$ there is a maximal e-rooted curve $\ell$ with $h(\ell)=p$ and $\bar{\omega}(c)=\omega(\mathcal{L}(\ell))$.

Assume that we apply Algorithm 1 on $T^{\prime}$ and that we currently consider the vertex $u$ of $T^{\prime}$. Hence, we can assume that for each vertex $v \neq u$ of $T_{u}^{\prime}$ its optimal candidate and $\omega(\mathcal{L}(v))$ is given. We first explain how to speed up OptCandidate using linearizations. Afterwards, we present the construction of linearizations.

Application of linearizations. Here we assume that the linearizations are given for the edges of $T$. Concerning the type of $u$ we describe how to compute its optimal candidate.

Case 1, $u$ is regular. If $u$ is a leaf, the set $C(u)$ contains only $\perp_{u}$. Hence, assume that $u$ has one outgoing edge $e=(u, v) \in E^{\prime}$, which belongs to a road $R$. Let $P$ be the longest path of vertices in $T_{u}^{\prime}$ that starts at $u$ and does not contain any junction vertex. Note that the path must be unique. Further, by construction of $T^{\prime}$ the last vertex $w$ of $P$ must be a regular vertex in $V$, but not in $V^{\prime} \backslash V$. We consider two cases; see Fig 7

If $\mathrm{d}(u, w) \geq \lambda(R)$, the optimal candidate is either $\perp_{u}$ or

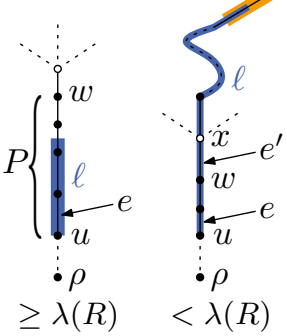

Fig. 7. Case 1 the $e$-rooted curve $\ell$ of length $\lambda(R)$ that ends on $\mathrm{E}(P)$. By assumption and due to $\omega\left(\mathcal{L}\left(\perp_{u}\right)\right)=\omega(\mathcal{L}(v))$, we decide in $O(1)$ time whether $\omega\left(\mathcal{L}\left(\perp_{u}\right)\right) \geq \omega(\mathcal{L}(\ell))$, obtaining the optimal candidate.

If $\mathrm{d}(u, w)<\lambda(R)$, the optimal candidate is either $\perp_{u}$ or goes through a junction. Since $w$ is regular, it has only one outgoing edge $e^{\prime}=(w, x)$. Further, by the choice of $P$ the edge $e^{\prime}$ is a junction edge in $T$; therefore the linearization $(L, \bar{\omega})$ of $e^{\prime}$ is given. In linear time we search for the curve $c \in L$ such that there is an $e$-rooted curve $\ell$ of length $\lambda(R)$ with its head on $c$. To that end we consider for each curve $c \in L$ its distance interval $I(c)$ and check whether there is $t \in I(c)$ with $t-\mathrm{d}_{u}=\lambda(R)$. Note that using a binary search tree for finding $c$ speeds this procedure up to $O(\log n)$ time, however, this does not asymptotically improve the total running time. The $e$-rooted curve $\ell$ then can be easily constructed in $O(n)$ time by walking from $c$ to $u$ in $\mathrm{E}(T)$.

If such a curve $c$ exist, by definition of a linearization the optimal candidate is either $\perp_{u}$ or $\ell$, which we can decide in $O(1)$ time by checking $\omega\left(\mathcal{L}\left(\perp_{u}\right)\right) \geq \omega(\mathcal{L}(\ell))$.

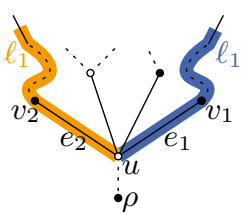

Fig. 8. Case 2 Note that we have $\omega\left(\mathcal{L}\left(\perp_{u}\right)\right)=\omega(\mathcal{L}(v))$ and $\omega(\mathcal{L}(\ell))=\bar{\omega}(c)$. If $c$ does not exist, again by definition of a linearization there is no vertical label $\ell \in C(u)$ and $\perp_{u}$ is the optimal candidate.

Case $2, u$ is a junction vertex. The set $C(u)$ contains horizontal labels. Let $\ell$ be such a label and let $e_{1}=\left(u, v_{1}\right)$ and $e_{2}=\left(u, v_{2}\right)$ be two junction edges in $E$ covered by $\ell$; see Fig. 8 Then there is an $e_{1}$-rooted curve $\ell_{1}$ and an $e_{2}$ rooted curve $\ell_{2}$ whose composition is $\ell$. Further, we have $\omega(\mathcal{L}(\ell))=\omega\left(\mathcal{L}\left(\ell_{1}\right) \cup \mathcal{L}\left(\ell_{2}\right)\right)+\sum_{v \in N(u) \backslash\left\{v_{1}, v_{2}\right\}} \omega(\mathcal{L}(v))$. We use this as follows.

Let $e_{1}$ and $e_{2}$ be two outgoing edges of $u$ that belong to the same road $R$, and let $\left(L_{1}, \bar{\omega}_{1}\right)$ and $\left(L_{2}, \bar{\omega}_{2}\right)$ be the linearizations of $e_{1}$ and $e_{2}$, respectively. We define for $e_{1}$ 
and $e_{2}$ and their linearizations the operation opt-cand $\left(L_{1}, L_{2}\right)$ that finds an optimal candidate of $u$ restricted to labels identifying $e_{1}$ and $e_{2}$.

For $i=1,2$ let $d_{i}=\max \left\{\mathrm{d}_{u} \mid u\right.$ is vertex of $\left.T_{v_{i}}\right\}$ and let $f_{u}(t)=\mathrm{d}_{u}-\left(t-\mathrm{d}_{u}\right)=$ $2 \mathrm{~d}_{u}-t$ be the function that "mirrors" the point $t \in \mathbb{R}^{2}$ at $\mathrm{d}_{u}$.

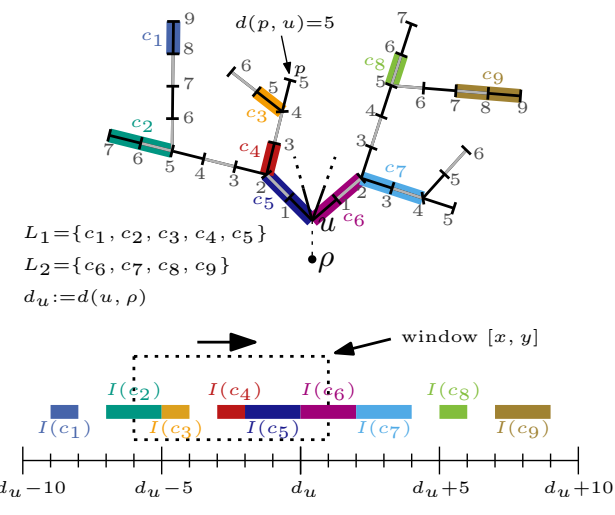

Fig. 9. Constructing the optimal candidate of $u$ based on the linearizations $\left(L_{1}, \bar{\omega}_{1}\right)$ and $\left(L_{2}, \bar{\omega}_{2}\right)$. The tree is annotated with distance marks.
Applying $f_{u}(t)$ on the boundaries of the distance intervals of the curves in $L_{1}$, we first mirror these intervals such that they are contained in the interval $\left[2 \mathrm{~d}_{u}-d_{1}, \mathrm{~d}_{u}\right]$; see Fig. 9. Thus, the curves in $L_{1} \cup L_{2}$ are mutually superposition-free such that their distance intervals lie in $J=\left[2 \mathrm{~d}_{u}-d_{1}, d_{2}\right]$. We call an interval $[x, y] \subseteq J$ a window, if it has length $\lambda(R), \mathrm{d}_{u} \in[x, y]$ and there are curves $c_{1} \in L_{1}$ and $c_{2} \in$ $L_{2}$ with $x \in I\left(c_{1}\right)$ and $y \in I\left(c_{2}\right)$; see Fig. 9. By the definition of a linearization there is a maximal $e_{1}$-rooted curve $\ell_{1}$ ending on $c_{1}$ and a maximal $e_{2}$-rooted curve $\ell_{2}$ ending on $c_{2}$ such that $\operatorname{len}\left(\ell_{1}\right)+\operatorname{len}\left(\ell_{2}\right)=\lambda(R)$. Consequently, the composition of $\ell_{1}$ and $\ell_{2}$ forms a horizontal label $\ell$ with $\omega(\mathcal{L}(\ell))=$ $\omega\left(\mathcal{L}\left(\ell_{1}\right) \cup \mathcal{L}\left(\ell_{2}\right)\right)+\sum_{v \in N(u) \backslash\left\{v_{1}, v_{2}\right\}} \mathcal{L}(v)$; we call $\omega(\mathcal{L}(\ell))$ the value of the window. Using a simple sweep from left to right we compute for the distance interval $I(c)$ of each curve $c \in L_{1} \cup L_{2}$ a window $[x, y]$ that starts or ends in $I(c)$ (if such a window exists). The result of opt-cand $\left(L_{1}, L_{2}\right)$ is then the label $\ell$ of the window with maximum value. For each pair $e_{1}$ and $e_{2}$ of outgoing edges we apply opt-cand $\left(L_{1}, L_{2}\right)$ computing a label $\ell$. By construction either the label $\ell$ with maximum $\omega(\ell)$ or $\perp_{u}$ is the optimal candidate for $u$, which we can check in $O(1)$ time. Later on we prove that we consider only linearizations of linear size. Since each vertex of $T^{\prime}$ has constant degree, we obtain the next lemma.

Lemma 3. For each $u \in V^{\prime}$ the optimal candidate can be found in $O(n)$ time.

Construction of linearizations. We now show how to recursively construct a linearization for an edge $e=(u, v)$ of $T$. To that end we assume that we are given the subdivision tree $T^{\prime}$ of $T$ and the linearizations for the outgoing edges $e_{1}=\left(v, w_{1}\right), \ldots, e_{k}=\left(v, w_{k}\right)$ of $v$ that belong to the same road $R$ as $e$. Further, we can assume that we have computed the weight $\omega(\mathcal{L}(w))$ for all vertices $w$ in $T_{u}^{\prime}$ excluding $u$. In case that two vertices of those vertices share the same position in $\mathrm{E}\left(T_{u}^{\prime}\right)$ we remove that one with less weight. Let $T_{i}$ be the tree induced by the edges $e, e_{i}$ and the edges of the subtree rooted at $w_{i}$. As a first step we compute for each linearization $(L, \bar{\omega})$ of each edge $e_{i}$ a linearization $\left(L_{i}, \bar{\omega}_{i}\right)$ for $e$ restricted to tree $T_{i}$, i.e., conceptually, we assume that $T_{u}$ only consists of $T_{i}$ 's edges. 


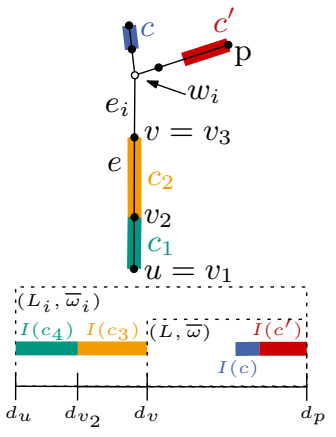

Fig. 10. 1st Step: For each edge $e_{i}$ extend its linearization $(L, \bar{\omega})$ to a linearization $\left(L_{i}, \bar{\omega}_{i}\right)$ of $T_{i}$.

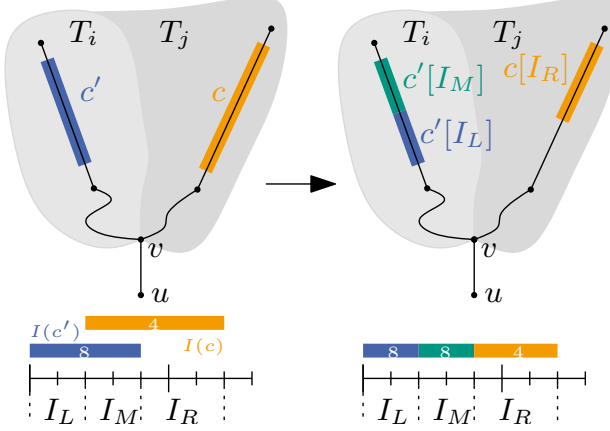

Fig. 11. 2nd Step: Merging the linearizations of the trees $T_{i}$ and $T_{j}$.

If $e$ is a junction edge we set $L_{i} \leftarrow L$ and weight each curve $c \in L_{i}$ as follows.

$$
\bar{\omega}_{i}(c) \leftarrow \bar{\omega}(c)+\sum_{w \in N(v) \backslash\left\{w_{i}\right\}} \omega(\mathcal{L}(w))
$$

Otherwise, if $e$ is a road section, let $v_{1}, \ldots, v_{l}$ be the vertices of the subdivision tree $T^{\prime}$ that lie on $e$, i.e., $\mathrm{E}\left(v_{j}\right) \in \mathrm{E}(e)$ for all $1 \leq j \leq l$; see Fig. 10 . We assume that $\mathrm{d}\left(v_{1}\right)<\ldots<\mathrm{d}\left(v_{l}\right)$, which in particular yields $v_{1}=u$ and $v_{l}=v$. Let $c_{1}$ be the curve $\mathrm{E}\left(\left(v_{1}, v_{2}\right)\right)$ and for $2 \leq j<l$ let $c_{j}$ be the curve $\mathrm{E}\left(\left(v_{j}, v_{j+1}\right)\right) \backslash \mathrm{E}\left(v_{j}\right)$. Hence, we have $\bigcup_{j=1}^{l} c_{j}=\mathrm{E}(e)$ and $c_{j} \cap c_{j^{\prime}}=\emptyset$ for $1 \leq j<j^{\prime}<l$. We set

$$
L_{i} \leftarrow L \cup \bigcup_{j=1}^{l-1}\left\{c_{j}\right\}
$$

We weight each curve $c \in L_{i}$ as follows. If $c$ is contained in $L$, we set

$$
\bar{\omega}_{i}(c) \leftarrow \bar{\omega}(c)+1
$$

Otherwise, $c$ is a sub-curve of $\mathrm{E}(e)$ and there exists a $j$ with $c=c_{j}$. We set

$$
\bar{\omega}_{i}(c) \leftarrow \omega\left(\mathcal{L}\left(v_{j+1}\right) \cup\left\{\ell_{c}\right\}\right),
$$

where $\ell_{c} \subseteq \mathrm{E}(e)$ is an $e$-rooted curve that starts at $\mathrm{E}(u)$ and ends on $c$. The next lemma shows that this transformation yields a linearization as desired.

Lemma 4. For each outgoing edge $e_{i}$ with linearization $(L, \bar{\omega})$ the tuple $\left(L_{i}, \bar{\omega}_{i}\right)$ is a linearization of e restricted to the tree $T_{i}$.

Proof. We use the same notation as used above.

First of all, the set $L_{i}$ contains only curves that do not superpose each other: Since $L_{i}$ contains only curves that do not superpose each other, the only curves that could 
superpose another curve in $L$ are contained in $L_{i} \backslash L$. Since $L_{i} \backslash L$ is empty for a junction edge, we can assume that $e$ is a road section. By construction those curves in $L_{i} \backslash L$ partition $\mathrm{E}(e)$ without intersecting each other. Further, by assumption no two road sections share a common vertex and since all curves of $L$ are contained in $\mathrm{E}\left(T_{v}\right)$, the curves in $L_{i} \backslash L$ cannot superpose any curve in $L$.

We now prove that $L_{i}$ satisfies the three conditions of a linearization. First assume that $e$ is a road section.

Condition (17). Since $L$ is a linearization, each curve of $L$ must be a sub-curve of a road section. Further, the curves $L_{i} \backslash L$ are sub-curves of the road section $e$.

Condition (2). First consider an $e$-rooted curve $\ell$ that either ends on $e_{i}$ or on an edge of $T_{w_{i}}$. Recall that $h(\ell)$ must lie on a road section. Then there is an $e_{i}$-rooted curve $\ell^{\prime}$ with $\ell^{\prime} \subseteq \ell$ and $h(\ell)=h\left(\ell^{\prime}\right)$. Hence, there is a curve $c \in L$ with len $\left(\ell^{\prime}\right)+\mathrm{d}_{v} \in I(c)$. Since $\ell^{\prime}$ is a sub-curve of $\ell$, we also have $\operatorname{len}(\ell)+\mathrm{d}_{u} \in I(c)$. Now, consider an $e$-rooted curve $\ell$ that ends on $e$, then obviously by construction there is a curve $c \in L_{i} \backslash L$ with $\operatorname{len}(\ell)+\mathrm{d}_{u} \in I(c)$.

Condition (3). First consider an arbitrary curve $c \in L_{i} \backslash L$ and let $\ell$ be any $e$-rooted curve that ends on $c$. Further, let $v_{1}, \ldots, v_{l}$ be the vertices of the subdivision tree $T^{\prime}$ that lie on $e$ as defined above. By construction there is an edge $\left(v_{j}, v_{j+1}\right)$ with $1 \leq j<l$ and $c \subseteq \mathrm{E}\left(v_{j}, v_{j+1}\right)$. It holds

$$
\omega(\mathcal{L}(\ell))=\omega\left(\mathcal{L}\left(v_{j+1}\right) \cup\{\ell\}\right)=\bar{\omega}_{i}(c)
$$

Obviously, $\ell$ must be maximal, because there is no other point in $\mathrm{E}\left(T_{i}\right)$ having the same distance to $\rho$ as $h(\ell)$ has.

Finally, consider a curve $c \in L$ and let $\ell$ be any $e$-rooted curve that ends on $c$. As $L$ is a linearization of $e_{i}$, for each point $p$ on $c$ there must be an $e_{i}$-rooted curve $\ell^{\prime}$ with $h\left(\ell^{\prime}\right) \in c$. We choose $\ell^{\prime}$ such that $h\left(\ell^{\prime}\right)=h(\ell)$. Since $\ell^{\prime}$ is a maximal $e_{i}$-rooted curve, the curve $\ell$ must be a maximal $e$-rooted curve. Further, $\ell$ identifies one road section more than $\ell^{\prime}$. Hence, we obtain

$$
\omega(\mathcal{L}(\ell))=\omega\left(\mathcal{L}\left(\ell^{\prime}\right)\right)+1=\bar{\omega}(c)+1=\bar{\omega}_{i}(c)
$$

Now consider the case that $e$ is a junction edge. Condition (7) and Condition (2) follow by the same arguments as stated above with the simplification that $L_{i}=L$.

Condition (3). Let $c$ be a curve in $L_{i}$ and let $\ell$ be any $e$-rooted curve that ends on $c$. Further, let $\ell^{\prime}$ be the $e_{i}$-rooted sub-curve of $\ell$ that starts at $\mathrm{E}(v)$ and ends at $h(\ell)$; by definition of $L$ such a curve exists. It holds

$$
\omega(\mathcal{L}(\ell))=\omega\left(\mathcal{L}\left(\ell^{\prime}\right)\right)+\sum_{w \in N(v) \backslash\left\{w_{i}\right\}} \omega(\mathcal{L}(w))=\bar{\omega}(c)+\sum_{w \in N(v) \backslash\left\{w_{i}\right\}} \omega(\mathcal{L}(w))=\bar{\omega}_{i}(c)
$$

Since $\ell^{\prime}$ is a maximal $e_{i}$-rooted curve, it directly follows that $\ell$ is a maximal $e$-rooted curve with respect to $T_{i}$.

In the next step we define an operation $\oplus$ by means of which two linearizations $\left(L_{i}, \bar{\omega}_{i}\right)$ and $\left(L_{j}, \bar{\omega}_{j}\right)$ can be combined to one linearization $\left(L_{i}, \bar{\omega}_{i}\right) \oplus\left(L_{j}, \bar{\omega}_{j}\right)$ of $e$ that is restricted to the subtree $T_{i, j}$ induced by the edges of $T_{i}$ and $T_{j}$. Consequently, $\bigoplus_{i=1}^{k}\left(L_{i}, \bar{\omega}_{i}\right)$ is the linearization of $e$ without any restrictions. 


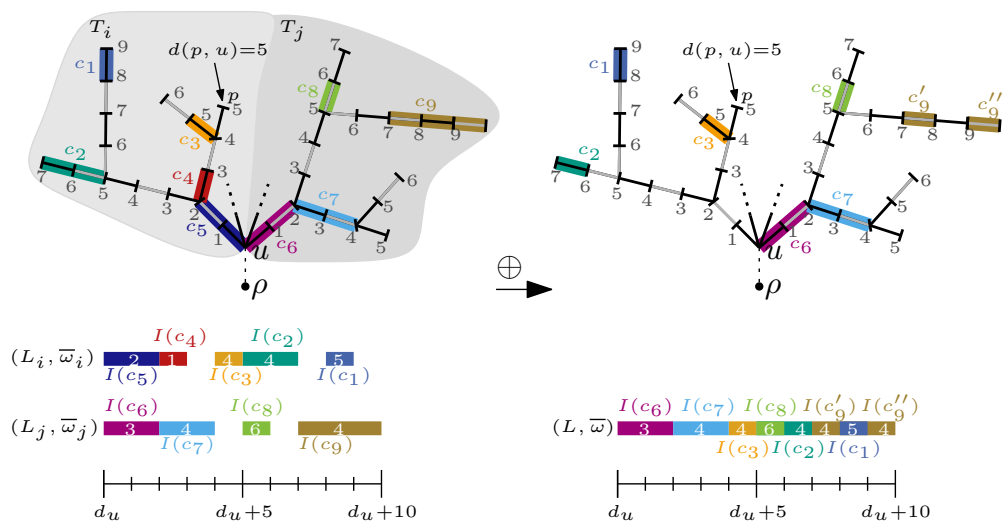

Fig. 12. Illustration of merging two linearizations $\left(L_{i}, \bar{\omega}_{i}\right)$ and $\left(L_{j}, \bar{\omega}_{j}\right)$ into one linearization $\left(L_{1}, \bar{\omega}_{i}\right)$. The trees are annotated with distance marks.

We define $(L, \bar{\omega})=\left(L_{i}, \bar{\omega}_{i}\right) \oplus\left(L_{j}, \bar{\omega}_{j}\right)$ as follows; for illustration see also Fig. 12 . Let $c_{1}, \ldots, c_{\ell}$ be the curves of $L_{i} \cup L_{j}$ such that for any two curves $c_{s}, c_{t}$ with $s<t$ the left endpoint of $I\left(c_{s}\right)$ lies to the left of the left endpoint of $I\left(c_{t}\right)$; ties are broken arbitrarily. We successively add the curves to $L$ in the given order enforcing that the curves in $L$ remain superposition-free. Let $c$ be the next curve to be added to $L$.

Without loss of generality, let $c \in L_{i}$. The opposite case can be handled analogously. In case that there is no curve superposing $c$, we add $c$ to $L$ and set $\bar{\omega}(c)=\bar{\omega}_{i}(c)$. If $c$ superposes a curve in $L$, due the order of insertion, there can only be one curve $c^{\prime}$ in $L$ that superposes $c$. First we remove $c^{\prime}$ from $L$. Let $I_{M}$ be the interval describing the set $I(c) \cap I\left(c^{\prime}\right)$, and let $I_{L}$ and $I_{R}$ be the intervals describing the set $I(c) \cup I\left(c^{\prime}\right) \backslash\left(I(c) \cap I\left(c^{\prime}\right)\right)$ such that $I_{L}$ lies to the left of $I_{M}$ and $I_{R}$ lies to the right of $I_{M}$; see Fig. 11 .

We now define three curves $c_{L}, c_{M}$ and $c_{R}$ with $I\left(c_{L}\right)=I_{L}, I\left(c_{M}\right)=I_{M}$ and $I\left(c_{R}\right)=I_{R}$ such that each of these three curves is a sub-curve of either $c$ or $c^{\prime}$. To that end let $c[I]$ denote the sub-curve of $c$ whose distance interval is $I$. We define the curve $c_{R}$ with weight $\bar{\omega}\left(c_{R}\right)$ as

$$
\left(c_{R}, \bar{\omega}\left(c_{R}\right)\right)= \begin{cases}\left(c\left[I_{R}\right], \bar{\omega}_{i}(c)\right), & \text { if } I_{R} \subseteq I(c) \\ \left(c^{\prime}\left[I_{R}\right], \bar{\omega}\left(c^{\prime}\right)\right), & \text { if } I_{R} \subseteq I\left(c^{\prime}\right)\end{cases}
$$

The curve $c_{L}$ and its weight $\bar{\omega}\left(c_{L}\right)$ is defined analogously. The curve $c_{M}$ and its weight $\bar{\omega}\left(c_{M}\right)$ is

$$
\left(c_{M}, \bar{\omega}\left(c_{M}\right)\right)= \begin{cases}\left(c\left[I_{M}\right], \bar{\omega}_{i}(c)\right), & \text { if } \bar{\omega}_{i}(c) \geq \bar{\omega}\left(c^{\prime}\right) \\ \left(c^{\prime}\left[I_{M}\right], \bar{\omega}\left(c^{\prime}\right)\right), & \text { if } \bar{\omega}_{i}(c)<\bar{\omega}\left(c^{\prime}\right)\end{cases}
$$

The next lemma proves that $\left(L_{i}, \bar{\omega}_{i}\right) \oplus\left(L_{j}, \bar{\omega}_{j}\right)$ is a restricted linearization.

Lemma 5. Let $\left(L_{i}, \bar{\omega}_{i}\right)$ and $\left(L_{j}, \bar{\omega}_{j}\right)$ be two linearizations of $e=(u, v)$ that are restricted to the trees $T_{i}$ and $T_{j}$, respectively. Then $(L, \bar{\omega})=\left(L_{i}, \bar{\omega}_{i}\right) \oplus\left(L_{j}, \bar{\omega}_{j}\right)$ is a linearization of e restricted to $T_{i, j}$. The operation needs $O\left(\left|L_{i}\right|+\left|L_{j}\right|\right)$ time. 
Proof. First of all, the set $L$ contains only curves that are pairwise free from any superpositions. This directly follows from the construction that curves $c$ and $c^{\prime}$ superposing each other are replaced by three superposition-free curves $c_{L}, c_{M}$ and $c_{R}$. Due to $I\left(c_{L}\right) \cup I\left(c_{M}\right) \cup I\left(c_{R}\right)=I(c) \cup I\left(c^{\prime}\right)$ the first and second condition of a linearization is satisfied.

We finally prove that Condition (3) of a linearization is satisfied by doing an induction over the curves inserted to $L$. Let $L^{k}$ be $L$ after the $k$-th insertion step. Since $L^{0}$ is empty, the condition obviously holds for $L^{0}$. So assume that we insert $c$ to $L^{k}$ obtaining the set $L^{k+1}$. Without loss of generality assume that $c \in L_{i}$. If $c$ does not superpose any curve in $L^{k}$, the condition directly follows from the definition of $c$. So assume that $c^{\prime} \in L^{k}$ superposes $c$. Since $c \in L_{i}$, the curve $c^{\prime}$ is contained in $\mathrm{E}\left(T_{j}\right)$. We remove $c^{\prime}$ from $L^{k}$ and insert the curves $c_{R}, c_{M}$ and $c_{L}$ as defined above. We prove that all three curves satisfy Condition (3).

Consider in the following the subtree $T_{i, j}$ of $T_{u}$ restricted to the edges of $T_{i}$ and $T_{j}$. We set $c_{R}=c\left[I_{R}\right]$ and set $\bar{\omega}\left(c_{R}\right)=\bar{\omega}_{i}(c)$, if $I_{R} \subseteq I(c)$. In that case there is no $e$-rooted curve $\ell \subseteq \mathrm{E}\left(T_{j}\right)$ with $\operatorname{len}(\ell)+\mathrm{d}_{u} \in I_{R}$, i.e., either there is no curve $\ell$ in $\mathrm{E}\left(T_{j}\right)$ with $t(\ell)=\mathrm{E}(u)$ and len $(\ell)+\mathrm{d}_{u} \in I_{R}$, or any curve in $\mathrm{E}\left(T_{j}\right)$ with $t(\ell)=\mathrm{E}(u)$ and len $(\ell)+\mathrm{d}_{u} \in I_{R}$ ends on a junction edge. Consequently, any $e$-rooted curve $\ell$ with len $(\ell)+\mathrm{d}_{u} \in I_{R}$ and in particular any maximal $e$-rooted curve $\ell$ with len $(\ell)+\mathrm{d}_{u} \in$ $I_{R}$ lies in $\mathrm{E}\left(T_{i}\right)$. Thus, the curve $c_{R}$ satisfies Condition (3). For the case $I_{R} \subseteq I\left(c^{\prime}\right)$ and the curve $c_{L}$ we can argue analogously.

So consider the curve $c_{M}$. Without loss of generality we assume that $\bar{\omega}_{i}(c) \geq \bar{\omega}\left(c^{\prime}\right)$. The opposite case can be handled analogously. For any maximal $e$-rooted curve $\ell$ in $\mathrm{E}\left(T_{j}\right)$ with len $(\ell)+\mathrm{d}_{u} \in I_{M}$ it must be true that $\omega(\mathcal{L}(\ell)) \leq \bar{\omega}\left(c_{M}\right)$. Further, since $c_{M} \subseteq c$ and $c$ satisfies condition (3) with respect to $T_{i}, c_{M}$ satisfies the condition (3) with respect to $T_{i, j}$.

Lemma 4 and Lemma 5 yield that $\bigoplus_{i=1}^{k}\left(L_{i}, \omega_{i}\right)$ is the linearization of $e$ without any restrictions. Computing it needs $O\left(\sum_{i=1}^{k}\left|L_{i}\right|\right)$ time.

Note that when computing optimal candidates (see Application of linearizations) we are only interested in $e$-rooted curves $\ell$ that have length at most $\lambda(R)$, where $R$ is the road of $e$. Hence, when constructing $\left(L_{i}, \bar{\omega}_{i}\right)$ for an edge $e_{i}$ in the first step, we discard any curve $c$ of $L_{i}$ that does not allow an $e$-rooted curve that both ends on $c$ and has length at most $\lambda(R)$; the curve $c$ is not necessary for our purposes. Hence, we conceptually restrict $T_{i}$ to the edges that are reachable from $u$ by one label length. It is not hard to see that $T^{\prime}$ restricted to $\mathrm{E}\left(T_{i}\right)$ contains only $O(n)$ vertices, because each vertex of $V^{\prime} \backslash V$ is induced by a chain of tightly packed vertical labels, whereas each label has length $\lambda(R)$. Hence, $T^{\prime}$ restricted to $\mathrm{E}\left(T_{i}\right)$ contains for each such chain at most one vertex of $V^{\prime} \backslash V$. Further, the endpoints of the curves in $L_{i}$ are induced by the vertices of $T^{\prime}$. Hence, by discarding the unnecessary curves of $L_{i}$ the set $L_{i}$ has size $O(n)$. Altogether, by Lemma 5 and due to the constant degree of each vertex we can construct $\bigoplus_{i=1}^{k}\left(L_{i}, \omega_{i}\right)$ in $O\left(\sum_{i=1}^{k} n\right)=O(n)$ time.

When constructing $\mathcal{L}(u)$ for $u$ as described in Algorithm 1 , we first build the linearization $L_{e}$ of each of $u$ 's outgoing edges. By Lemma 3 we can find in $O(n)$ time the optimal candidate of $u$. Then, due to the previous reasoning, the linearization of an edge 
of $T$ and the optimal candidate of a vertex $u$ can be constructed in $O(n)$ time. Altogether we obtain the following result.

Proposition 1. For a road map $\mathcal{M}$ with a tree $T$ as underlying road graph, MAXIDENTIFIEDROADS can be solved in $O\left(n^{3}\right)$ time.

\subsection{Improvements on Storage Consumption}

Since $T^{\prime}$ contains $O\left(n^{2}\right)$ vertices, the algorithm needs $O\left(n^{2}\right)$ space. This can be improved to $O(n)$ space. To that end $T^{\prime}$ is constructed on the fly while executing Algorithm 1. Parts of $T^{\prime}$ that become unnecessary are discarded. We prove that it is sufficient to store $O(n)$ vertices of $T^{\prime}$ at any time such that the optimal labeling can still be constructed.

When constructing the optimal labeling of $T$, we build for each edge $(u, v)$ of $T$ its linearization based on the linearization of the outgoing edges of $v$. Afterwards we discard the linearizations of those outgoing edges. Since each vertex has constant degree, considering

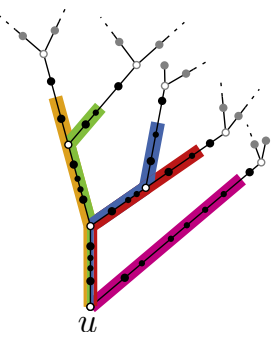

Fig. 13. Vertices not reachable from $u$ are marked gray. such that $\mathrm{E}(v) \in \ell$ or $v \in N(\ell)$, where len $(\ell)$ denotes the length of $\ell$; see Fig. 13 . The set $\mathrm{R}_{u}$ contains all vertices of $T_{u}^{\prime}$ that are reachable from $u$. The next lemma shows that $\mathrm{R}_{u}$ has linear size. 
Lemma 6. For any vertex $u$ of $T^{\prime}$ the set $\mathrm{R}_{u}$ has size $O(n)$.

Proof. Recall how $T^{\prime}$ is constructed: For each vertex $v \in V$ we construct a chain $C$ of tightly packed vertical valid labels, which starts at $\mathrm{E}(v)$, is directed towards $\rho$, and ends when either the road ends, or adding the next label does not increase the number of identified road sections. Each label of such a chain $C$ induces one vertex of $T^{\prime}$. Hence, $C$ induces a set $V_{C}$ of vertices in $T^{\prime}$. We show that for each chain $C$ the set $V_{C} \cap \mathrm{R}_{u}$ contains at most two vertices. As we construct $n$ chains in order to build $T^{\prime}$ the claim follows.

For the sake of contradiction assume that there is a chain $C$ and a vertex $u$ in $T^{\prime}$ such that $V_{C} \cap \mathrm{R}_{u}$ contains more than two vertices. Without loss of generality we assume that $V_{C} \cap \mathrm{R}_{u}$ contains three vertices, which we denote by $v_{1}$, $v_{2}$ and $v_{3}$. We further assume that $\mathrm{d}_{v_{1}}<\mathrm{d}_{v_{2}}<\mathrm{d}_{v_{3}}$. By construction all labels in $C$ lie in the embedding of the same road $R_{C}$, and $\mathrm{d}\left(v_{1}, v_{2}\right) \geq \lambda\left(R_{C}\right)$ and $\mathrm{d}\left(v_{2}, v_{3}\right) \geq \lambda\left(R_{C}\right)$. By definition of $C$ there is a vertical curve $\ell \in \mathrm{E}\left(T_{u}^{\prime}\right)$ that starts at $\mathrm{E}(u)$ and contains $v_{1}, v_{2}$ and $v_{3}$. Let $e$ be the outgoing edge of $u$ in $T^{\prime}$ whose embedding is covered by $\ell$ and consider the sub-curve $\ell^{\prime} \subseteq \ell$ with length $\lambda\left(R_{C}\right)$ that starts at $u$. By definition of $\mathrm{R}_{u}$, we know for each $v_{i}$ with $1 \leq i \leq 3$ that either its embbeding is contained in $\ell^{\prime}$ or $v_{i} \in N\left(\ell^{\prime}\right)$. From the definition of $N\left(\ell^{\prime}\right)$ and the fact that all three vertices lie on $\ell$, it directly follows that only $v_{3}$ may be contained in $N\left(\ell^{\prime}\right)$. Hence, $\mathrm{E}\left(v_{1}\right), \mathrm{E}\left(v_{2}\right) \in \ell^{\prime}$. Further, because $v_{2} \notin N\left(\ell^{\prime}\right)$, we have $\mathrm{E}\left(v_{2}\right) \neq h\left(\ell^{\prime}\right)$, which implies $\mathrm{d}\left(v_{1}, v_{2}\right)<\lambda(R)$ and contradicts $\mathrm{d}\left(v_{1}, v_{2}\right) \geq \lambda(R)$.

Assume that we apply Algorithm 1 considering the vertex $u$. When constructing $u$ 's optimal candidate, by Lemma 6 it is sufficient to consider the vertices of $T_{u}^{\prime}$ that lie in $\mathrm{R}_{u}$. On that account we discard all vertices of $T_{u}^{\prime}$ that lie in $V^{\prime} \backslash V$, but not in $\mathrm{R}_{u}$.

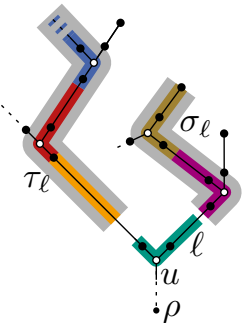

Fig. 14. Chains of label $\ell$.
Further, we compute the vertices of $V^{\prime} \backslash V$ that subdivide the incoming edge $(t, u) \in E$ on demand, i.e., we compute them, when constructing the optimal candidate of $t$. Hence, we have linear space consumption.

However, when discarding vertices of $T^{\prime}$, we lose the possibility of reconstructing the labeling. We therefore annotate each vertex $u \in V$ of the original tree $T$ with further information. To that end consider a canonical labeling $\mathcal{L}$ of $T$. Let $\ell$ be a horizontal label of $\mathcal{L}$ and let $e$ be the edge of $T$ on which $\ell$ 's head is located. Either, no other label of $\mathcal{L}$ ends on $e$, or another label $\ell^{\prime}$ ends on $e$ that belongs to a chain $\sigma_{\ell}$ of tightly packed vertical labels. Analogously, we can define the chain $\tau_{\ell}$ with respect to edge $e^{\prime}$ on which $\ell^{\prime} s$ tail is located. On that account we store for a junction vertex $u \in V$ not only its optimal candidate $\ell \in C(u)$, but also the two chains $\sigma_{\ell}$ and $\tau_{\ell}$, if they exist. Note that such a chain of tightly packed vertical labels is uniquely defined by its start and endpoint, which implies that $O(1)$ space is sufficient to store both chains. Using a breadth-first search we can easily reconstruct those chains in linear time. For a regular vertex $u \in V$ we analogously store $\sigma_{\ell}$ of its optimal candidate $\ell \in C(u)$, if it exists. Since $\ell$ is vertical, we do not need to consider its tail. For the special case that $\ell=\perp_{u}$, we define that $\sigma_{\ell}$ is the chain of tightly packed vertical labels that ends on the only 
outgoing edge $e$ of $u$. Summarizing, the additional information together with the optimal candidates stored at the vertices $u \in V$ of the original tree are sufficient to reconstruct the labeling of $T$. Together with Proposition 1 we obtain the following result.

Theorem 3. For a road map $\mathcal{M}$ with a tree $T$ as underlying road graph, MAXIDENTIFIEDROADS can be solved in $O\left(n^{3}\right)$ time using $O(n)$ space.

\section{On the Usefulness of Labeling Tree-Shaped Road Networks}

Although the underlying road graphs of real-world road maps are rarely trees, our algorithm for labeling trees is still of practical interest as we show in first initial experiments. The obtained data shall give the reader evidence of the practicability and relevance of our algorithm, but they are not yet a complete experimental study. For a companion paper we are working on a detailed evaluation of our approach and are investigating several practical heuristics that are based on the tree labeling algorithm.

To evaluate the usefulness of our algorithm we considered the road networks of several large cities. We extracted the road graphs from the data provided by OpenStreetMap ${ }^{1}$ and drew them mimicking the style used on openstreetmap.org as standard. In particular, we adapted the zoom level 17 , which maps $50 \mathrm{~m}$ to 65 pixels.

On each road graph we first applied a simple preprocessing strategy removing and cutting road sections that can be labeled trivially without violating any optimal solution. In particular we applied the following rules.

1. Remove any road that contains exactly one road section.

2. Remove any road section that is sufficiently long to completely contain a label and whose adjacent road sections are also sufficiently long to completely contain a label. Here two road sections are called adjacent, if they are connected by a path containing only junction edges.

3. Cut any road section into two halves that is sufficiently long to contain a label twice in a row.

That preprocessing strategy decomposed the road graphs into a large number of subgraphs; see Table 1 For example, for the road network of London, which consists of 143856 road sections, the rules of the preprocessing strategy matched 91405 road sections, so that the road graph decomposed into 21825 subgraphs. Note that if we are able to label those subgraphs optimally, we obtain an optimal labeling for the whole road network by the choice of the preprocessing rules. Table 1 further shows that most of those subgraphs are trees ( $85.1 \%$ for Berlin as a minimum and $97.7 \%$ for Los Angeles as a maximum). Hence, using our tree labeling algorithm we can label a large number of the remaining subgraphs optimally. We conjecture that using the preprocessing strategy in combination with the tree labeling algorithm and some heuristics or exact methods for the non-tree subgraphs we can label real-world instances near-optimally. This hypothesis is also supported by the observation that most of the road sections are either matched by the preprocessing strategy or are contained in trees $(55.7 \%+32.9 \%=88.6 \%$ for Paris as a minimum and $67.5 \%+28.6 \%=96.1 \%$ for Los Angeles as a maximum). For our planned companion paper we are currently working on corresponding experiments

1 openstreetmap.org 
Table 1. Number of connected subgraphs and road sections for road networks of five cities. The column subgraphs contains the number of connected subgraphs into which the graph is decomposed after preprocessing: 1 . the total number of subgraphs, 2. the number of trees, 3. the number of subgraphs with one cycle, and 4 . the number of subgraphs with more than one cycle. The column road sections contains the number of road sections 1 . in total, 2 . matched by the preprocessing strategy, 3. contained in trees, 4 . contained in subgraphs with one cycle and 5. contained in subgraphs with more than one cycle.

\begin{tabular}{|c|c|c|c|c|c|c|c|c|c|}
\hline \multirow{2}{*}{ Number of } & \multicolumn{4}{|c|}{ subgraphs (after preprocessing) } & \multicolumn{5}{|c|}{ road sections } \\
\hline & total & trees & 1 cycle & $\geq 2$ cycles & total & matched & trees & 1 cycle & $\geq 2$ cycles \\
\hline \multirow{2}{*}{ Berlin } & 5702 & 4853 & 549 & 300 & 49773 & 36021 & 8220 & 2170 & 3362 \\
\hline & $100 \%$ & $85.1 \%$ & $9.6 \%$ & $5.3 \%$ & $100 \%$ & $72.4 \%$ & $16.5 \%$ & $4.4 \%$ & $6.8 \%$ \\
\hline \multirow{2}{*}{ Paris } & 22929 & 20604 & 1742 & 583 & 145971 & 81305 & 48009 & 8329 & 8328 \\
\hline & $100 \%$ & $89.9 \%$ & $7.6 \%$ & $2.5 \%$ & $100 \%$ & $55.7 \%$ & $32.9 \%$ & $5.7 \%$ & $5.7 \%$ \\
\hline \multirow{2}{*}{ London } & 21825 & 20538 & 1012 & 275 & 143856 & 91405 & 44845 & 4485 & 3121 \\
\hline & $100 \%$ & $94.1 \%$ & $4.6 \%$ & $1.3 \%$ & $100 \%$ & $63.5 \%$ & $31.2 \%$ & $3.1 \%$ & $2.2 \%$ \\
\hline \multirow{2}{*}{ Los Angeles } & 48248 & 47131 & 767 & 350 & 397505 & 268334 & 113842 & 5149 & 10180 \\
\hline & $100 \%$ & $97.7 \%$ & $1.6 \%$ & $0.7 \%$ & $100 \%$ & $67.5 \%$ & $28.6 \%$ & $1.3 \%$ & $2.6 \%$ \\
\hline \multirow{2}{*}{ New York City } & 10318 & 9817 & 306 & 195 & 108417 & 72057 & 25549 & 3011 & 7800 \\
\hline & $100 \%$ & $95.1 \%$ & $3 \%$ & $1.9 \%$ & $100 \%$ & $66.5 \%$ & $23.6 \%$ & $2.8 \%$ & $7.2 \%$ \\
\hline
\end{tabular}

investigating that conjecture. Further, we are developing heuristics and exact algorithms for labeling the remaining non-tree subgraphs.

For example we can improve our results by adapting our tree labeling algorithm to subgraphs containing exactly one cycle $C$. We observe that there are three cases for such a subgraph: (1) no label identifies any road section of $C$, (2) there is a label $\ell$ that identifies only road sections of $C$, or (3) there is a label $\ell$ that identifies both road sections of $C$ and road sections of the remaining component. In the first case we can remove $C$ completely from the subgraph, such that it decomposes into a set of trees. In the second and third case the label $\ell$ splits the cycle $C$ so that the remaining road sections form trees. We explore all choices of $\ell$ taking the best choice. Hence, we can label subgraphs containing exactly one cycle optimally, which further increases the number of optimally labeled subgraphs ( $92.8 \%$ for New York City as a minimum and $97.8 \%$ for London as a maximum).

\section{Conclusions and Outlook}

In this paper we investigated the problem of maximizing the number of identified road sections in a labeling of a road map; we showed that it is NP-hard in general, but can be solved in $O\left(n^{3}\right)$ time and linear space for the special case of trees.

The underlying road graphs of real-world road maps are rarely trees. Initial experimental evidence indicates, however, that road maps can be decomposed into a large number of subgraphs by placing trivially optimal road labels and removing the cor- 
responding edges from the graph. It turns out that between $85.1 \%$ and $97.7 \%$ of the resulting subgraphs are actually trees, which we can label optimally by our proposed algorithm. As a consequence, this means that a large fraction (between $88.6 \%$ and $96.1 \%$ ) of all road sections in our real-world road graphs can be labeled optimally by combining this simple preprocessing strategy with the tree labeling algorithm. We are investigating further heuristic and exact approaches for labeling the remaining non-tree subgraphs (e.g., by finding suitable spanning trees and forests) for a separate companion paper. 


\section{References}

1. F. Chirié. Automated name placement with high cartographic quality: City street maps. Cartography and Geo. Inf. Science, 27(2):101-110, 2000.

2. S. Edmondson, J. Christensen, J. Marks, and S. M. Shieber. A general cartographic labelling algorithm. Cartographica, 33(4):13-24, 1996.

3. E. Imhof. Positioning names on maps. Amer. Cartogr., pages 128-144, 1975.

4. D. Lichtenstein. Planar formulae and their uses. SIAM J. Comput., 11(2):329-343, 1982.

5. S. Maass and J. Döllner. Embedded labels for line features in interactive 3d virtual environments. In Proc. 5th Int. Conf. Computer Graphics, Virtual Reality, Visualisation and Interaction in Africa, AFRIGRAPH '07, pages 53-59. ACM, 2007.

6. G. Neyer and F. Wagner. Labeling downtown. In Algorithms and Complexity (CIAC'OO), volume 1767 of $L N C S$, pages 113-124. Springer, 2000.

7. A. Reimer and M. Rylov. Point-feature lettering of high cartographic quality: A multi-criteria model with practical implementation. In EuroCG'14, Ein-Gedi, Israel, 2014.

8. S. Seibert and W. Unger. The hardness of placing street names in a Manhattan type map. Theor. Comp. Sci., 285:89-99, 2002.

9. T. Strijk. Geometric Algorithms for Cartographic Label Placement. Dissertation, Utrecht University, 2001.

10. M. Vaaraniemi, M. Treib, and R. Westermann. Temporally coherent real-time labeling of dynamic scenes. In Proc. 3rd Int. Conf. Comput. Geospatial Research Appl., COM.Geo '12, pages 17:1-17:10. ACM, 2012.

11. M. van Kreveld. Geographic information systems. In Handbook of Discrete and Computational Geometry, Second Edition, chapter 58, pages 1293-1314. CRC Press, 2010.

12. A. Wolff, L. Knipping, M. van Kreveld, T. Strijk, and P. K. Agarwal. A simple and efficient algorithm for high-quality line labeling. In Innovations in GIS VII: GeoComputation, chapter 11, pages 147-159. Taylor \& Francis, 2000.

13. A. Wolff and T. Strijk. The map labeling bibliography. http://liinwww.ira.uka de/bibliography/Theory/map.labeling.html 2009. 


\section{A Computational Complexity}

\section{A.1 Description of an Alternative Clause Gadget}

In this section we describe a clause gadget that can be used as an alternative to the one presented in Section 3 . Since it consists only of roads that are paths, this gadget strengthens Theorem 1 .

Theorem 4. For a given road map $\mathcal{M}$ and an integer $K$ it is NP-hard to decide if in total at least $K$ road sections can be identified, even if all roads are paths.

The clause gadget consists of ten roads, $r, g_{a}, g_{b}, g_{c}, a_{i}, b_{i}$ and $c_{i}$ with $i \in\{1,2\}$ that all are paths; see Fig. 15 Going along $r$ from one end to the other, the junctions with the roads $a_{i}, b_{i}$ and $c_{i}(1 \leq i \leq 2)$ occur in three densely packed blocks. The blocks are described by the sequence of roads intersecting $r$. The first block is $B_{a}=\left(a_{1}, c_{2}, b_{1}, a_{2}\right)$, the second block is $B_{b}=\left(a_{2}, b_{1}, c_{1}, b_{2}\right)$ and the third block is $B_{c}=\left(b_{2}, c_{1}, c_{2}, a_{1}\right)$. The label length of $r$ is chosen so that at most three labels can be placed on $r$, but each road section is shorter than a label of $r$. Choosing the length of the road sections appropriately, we further ensure that we can place a label that crosses all junctions of one of the blocks without crossing the junctions of another block.

We now describe junctions of the roads $g_{a}, g_{b}, g_{c}, a_{i}, b_{i}$ and $c_{i}$ with $i \in\{1,2\}$. The road $a_{1}$ first intersects $g_{a}$ and then $r$ twice. Let $s_{a_{1}}^{1}, s_{a_{1}}^{2}, s_{a_{1}}^{3}$ and $s_{a_{1}}^{4}$ denote these road sections in that particular order. The length of $s_{a_{1}}^{1}$ is chosen so that a single label can be placed on $s_{a_{1}}^{1}$, while the others are shorter than the label length of $a_{1}$. More specifically, we define $a_{1}$ 's label length such that a label identifies the sections in either $\left\{s_{a_{1}}^{1}\right\}$, $\left\{s_{a_{1}}^{1}, s_{a_{1}}^{2}\right\},\left\{s_{a_{1}}^{1}, s_{a_{1}}^{2}, s_{a_{1}}^{3}\right\},\left\{s_{a_{1}}^{2}, s_{a_{1}}^{3}, s_{a_{1}}^{4}\right\}$ or $\left\{s_{a_{1}}^{3}, s_{a_{1}}^{4}\right\}$. We define the intersections and the label length for $a_{2}$, analogously. Further, $g_{a}$ intersects $a_{1}$ and $a_{2}$ in one junction, i.e., the edge of $g_{a}$ connecting both junction vertices is a junction edge. The label length of $g_{a}$ is chosen so that a label can cross $g_{a}$ 's only junction. The length of $g_{a}$ 's road sections is at least as long as $g_{a}$ 's label length. We call $g_{a}$ a gate, because later this road will be connected to the end road of a chain by a junction; see violet square in Fig. 15(a) For $b_{1}, b_{2}, c_{1}, c_{2}$ we introduce analogous junctions and road sections, however, $b_{1}$ and $b_{2}$ intersect $g_{b}$ instead of $g_{a}$, and $c_{1}$ and $c_{2}$ intersect $g_{c}$ instead of $g_{a}$.

In order to identify both road sections of a gate, either two labels can be placed on the road sections separately, or one label that goes through the junction. In the former case the gate is open and in latter case it is closed; see Fig. 15(b). We observe that it only makes sense to close a gate, if at least one road section of the gate does not allow to place a label that is only contained in that road section. This case will occur if and only if the connected chain transmits the value false to the clause.

Assume that at least one gate is open, i.e., one literal of the clause is true; see Fig. 15(b) Without loss of generality let $g_{a}$ be open. We place a label $\ell_{r}$ on $r$ such that it crosses the junctions of block $B_{a}$ and identifies 5 sections. Since $g_{a}$ is open, we can place a label $\ell_{1}$ that identifies $s_{a_{1}}^{1}$ and $s_{a_{1}}^{2}$. Analogously, we can place a label $\ell_{2}$ identifying $s_{a_{2}}^{1}$ and $s_{a_{2}}^{2}$. Placing further labels as indicated in Fig. 15(b), we identify five road sections of $r$ and all road sections of any other road except for $s_{c_{2}}^{4}, s_{b_{1}}^{4}$. Hence, 33 road sections are identified. 


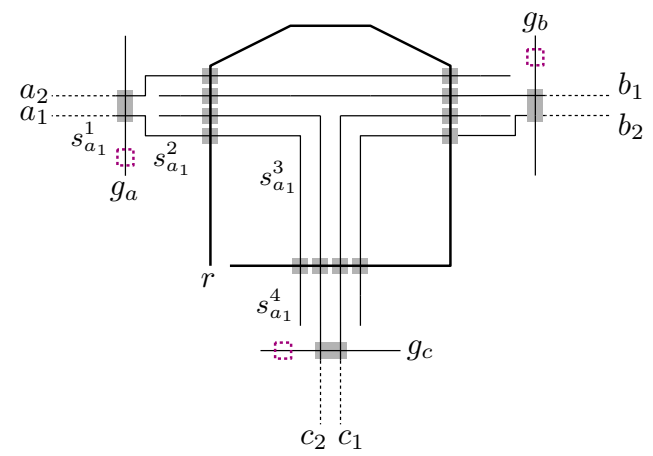

(a)

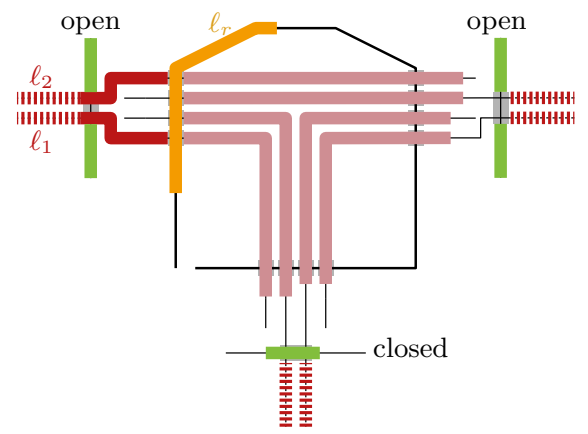

(b)

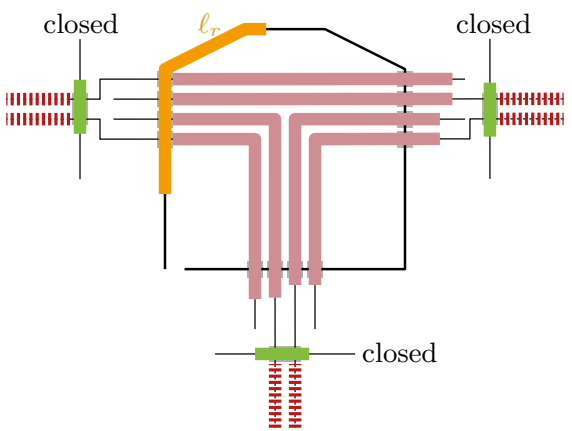

(c)

Fig. 15. Illustration of alternative clause gadget, which only uses paths as roads. (a) Structure of the clause gadget (b) Optimal labeleling for the case that at least one literal is true (c) Optimal labeling for the case that all literals are false.

We observe that we can place the labels of $b_{1}, b_{2}, c_{1}, c_{2}$ such that they do not cross the junctions of $g_{b}$ and $g_{c}$, respectively. Hence, it does not matter whether $g_{b}$ and $g_{c}$ are closed or open, i.e., it does not matter whether the corresponding literals are true or false.

We now argue that this is an optimal labeling. If $s_{c_{2}}^{4}$ or $s_{b_{1}}^{4}$ were labeled, the label $\ell_{r}$ must be placed such that the junctions of $r$ with $c_{2}$ and $b_{1}$ are not crossed, respectively. This decreases the number of identified road sections as least as much identifying $s_{c_{2}}^{4}$ and $s_{b_{1}}^{4}$ increases the number of identified road sections. In order to identify at least one of the unidentified road sections of $r$, we need to place a label that crosses $B_{b}$ or $B_{c}$. Obviously, this yields a smaller number of identified road sections than 31 .

Finally, assume that all gates are closed; see Fig. 15(c), Consider, the same labeling as before. However, this time we cannot label $s_{a_{1}}^{2}$ and $s_{a_{2}}^{2}$ anymore. Hence, this labeling has only 29 identified road sections. Obviously, it cannot be improved by changing the placement of the remaining labels or adding labels. 